\title{
TDOA Localization Algorithm based on Weight Fusion of Target Position Coordinates
}

\author{
Wu QingYi ${ }^{1,2, *}$, Zhang JianQiang ${ }^{1}$ and Sun KaiWen ${ }^{3}$ \\ ${ }^{I}$ College of Electronic Engineering, Naval University of Engineering, Wuhan 430000, China \\ ${ }^{2}$ College of naval aviation, Huludao 125000, China \\ ${ }^{3}$ Department of Computer Science and Engineering, University of California, San Diego, CA 92093, United States
}

Received 9 July 2017; Accepted 29 October 2017

\begin{abstract}
The use time of difference of arrival (TDOA) or angle of arrival (AOA) in locating the target requires at least four base stations. The measurement accuracy of different base stations varies due to different relative positions of the base station and the target. Therefore, the measurement results of different base stations cannot be discussed simultaneously. A new TDOA localization algorithm based on weighting fusion of position coordinates of target source was proposed to address this problem. First, maximum likelihood estimation was used to acquire the position coordinates of multiple groups of target sources on the 2D plane with time of arrival (TOA) and AOA of signals from base stations to the target source. Second, the coordinates were applied with weighted fusion according to the relative angle between the signals emitted from the base stations and target source to obtain relatively accurate 3D position coordinates. Finally, the proposed algorithm was compared with TDOA localization algorithm and TDOA-AOA combined localization algorithm. Results reveal that the proposed algorithm increases accuracy by $68.33 \%$ and $48.27 \%$ compared with TDOA localization algorithm and TDOA-AOA combined localization algorithm, respectively. Weighting data fusion can increase the calculation accuracy more effectively than the averaging method. This study increases the accuracy of 3D target localization in complicated environment. The proposed method shows prospect to optimize the wireless positioning technology in military and civilian fields.
\end{abstract}

Keywords: Wireless location, Time of difference of arrival, Maximum likelihood estimation, Weighted

\section{Introduction}

Wireless positioning technology plays an important role in military and civilian fields. Relevant technologies are one of the research hotspots in many fields. As a current popular localization technology, time difference of arrival (TDOA) determines the location of the moving target by detecting the time difference for the signal to arrive at different base stations. This method has been widely used in various fields [1-3].

TDOA localization technology is mainly applied to indoor high-accuracy wireless localization. This method can offer real-time localization of staff and equipment and has various functions, such as for track record, work attendance monitoring, behavioral analysis, and intelligent patrol [4-5]. However, the demand for outdoor remote localization increases gradually with the development of technologies and the growth of outdoor wireless localization demands. For example, wireless localization is needed to solve personnel and equipment tracking and localization in civilian fields, single solider positioning, projectile trajectory estimation, and battlefield situation estimation in military fields. The accurate and effective use of TDOA localization algorithm with high positioning accuracy and low calculation complexity is important in outdoor remote wireless localization.

\footnotetext{
*E-mail address: xioozhu054571@163.com

ISSN: 1791-2377 @ 2017 Eastern Macedonia and Thrace Institute of Technology. All rights reserved. doi:10.25103/iestr.106.05
}

TDOA algorithm localizes the target by solving the ternary equations, which were set up by measuring the time of arrival (TOA) of signals from the base stations to the target source. Given that outdoor environment is more complicated, changing, and has more interference factors of positioning accuracy than indoor environment, the TDOA algorithm must be improved in outdoor environment to reduce measurement error and improve the accuracy of the algorithm. The number of base stations can be increased, or TDOA algorithm can be combined with other localization techniques to increase the positioning accuracy [6-7]. However, the calculation load increases with the increasing number of base stations. Under this circumstance, the complicated 3D problem can be transformed into a simple 2D problem by projecting the base stations and target source onto the 2D plane. The binary equation system is created, and multiple groups of target coordinates are acquired. However, integrating the multiple groups of target coordinates to obtain accurate localization outcomes is still a problem that must be solved urgently.

This study proposed a new TDOA algorithm based on the weighting fusion of position coordinates of target source. This algorithm integrates multiple groups of coordinates and increases the positioning accuracy more effectively than the averaging method.

\section{State of the art}

TODA localization algorithm is often combined with other wireless positioning technologies to increase the positioning 
accuracy. C Liu [8] simply combined TDOA algorithm and AOA algorithm and achieved localization by calculating the azimuth angle and radial distance. This TDOA-AOA combined algorithm localizes the target by setting only three base stations but neglects the damages and does not analyze the possible measurement error caused by time difference and angle. X Zhang and ST Sihong [9] introduced one auxiliary variable and designed a new integrating closedloop fusion (ICF) algorithm. This ICF algorithm was applicable for the 3D positioning of TDOA, AOA, and DOA and for any sensor network distribution but not for any situation with only two base stations. Giacometti [10] increased the positioning accuracy by reflecting TDOA and AOA results using the specified emitter, which, however, increased the difficulty of engineering practice. Shikur [11, 12] and Giacometti and Baussard [13] combined TDOA algorithm and AOA algorithm and effectively increased the positioning accuracy. However, this TDOA-AOA combined algorithm requires calculating the Cramér-Rao lower bound (CRLB) and shows high complexity. Lim and Kang [14, 15] proposed vertical position estimation based on AOA localization algorithm and horizontal position estimation based on TDOA localization algorithm. This method could achieve high 3D positioning accuracy but does not consider the measurement errors caused by the environment of the equipment. Lei Wen-ying [16] first measured the angle and distance between the beacon node and the target and then calculated the coordinates of the target source accuracy. This algorithm has low complexity and could accomplish computation by three beacon nodes of only one base station during signal transmission. However, this method also neglects the damages. Kim, Choi, Alarifi and Anumba [1720] estimated the error when applying TDOA localization algorithm by using the Kalman filtering, which increased the positioning accuracy but complicated the computation. Tang and Park [21-22] implemented target localization by geometric method, but the localization region was limited.

These methods have high computation complexity when too many base stations are used. By contrast, localization algorithms with simple computation have limited number of base stations. None of these methods can effectively solve the error caused by the damages on base stations. In this study, the position coordinates of multiple groups of target sources on the 2D plane were acquired by using the maximum likelihood estimation with TOA and AOA of signals from the base stations to the target source. Weighting fusion was conducted for the acquired position coordinates according to the relative angle between the signals emitted from the base stations and target source. The proposed algorithm effectively relieves the heavy calculation load caused by the involvement of many base stations and guarantees accurate target positioning.

The remainder of this study is organized as follows. Section 3 mainly describes transforming the target localization problem in a $3 \mathrm{D}$ space into the localization problem in a $2 \mathrm{D}$ plane. The $2 \mathrm{D}$ position coordinates are acquired by the maximum likelihood method, and all data are fused by the weighting method to obtain relatively accurate positioning results. Section 4 presents the comparison of the proposed algorithm with pure TDOA algorithm and TDOA-AOA combined algorithm to verify the accuracy and feasibility of the proposed algorithm. Section 5 summarizes the conclusions.

\section{Methodology}

\subsection{D plane coordinates of the target source}

A $3 \mathrm{D}$ coordinate plane $(X, Y, Z)$ is constructed, and base stations are set up in the coordinate plane. The position coordinates of the reference base station are $\left(x_{0}, y_{0}, z_{0}\right)$, whereas those of the base station $i$ are $\left(x_{i}, y_{i}, z_{i}\right),(1 \leq i \leq n)$. The coordinates of target source $T$ are $\left(x_{T}, y_{T}, z_{T}\right)$, and the time for the target source to receive signals from the base station $i$ is $t_{i}$. The distance $d_{i}$ between the base stations and target source could be calculated based on the TDOA algorithm. Specific methods are expressed as follows [2324]:

$$
\left\{\begin{array}{c}
d_{1}=c \times\left(t_{1}-t_{0}\right) \\
d_{2}=c \times\left(t_{2}-t_{0}\right) \\
\vdots \\
d_{n}=c \times\left(t_{n}-t_{0}\right)
\end{array}\right.
$$

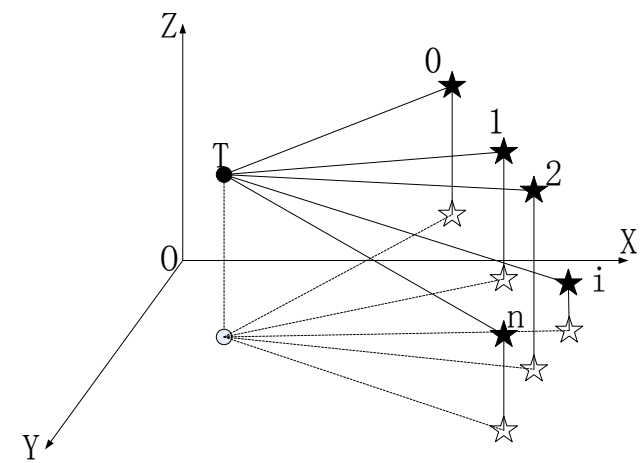

Fig. 1. Projections of the target source and base station in the XY plane

In Fig.1, the base stations and target source are projected onto the 2D XY plane named as $\alpha$ plane. The projection coordinates of the base stations and target source are $\left(x_{\alpha i}, y_{\alpha i}\right),(1 \leq i \leq n)$, and $\left(x_{\alpha T}, y_{\alpha T}\right)$. The angle of pitch of signals emitted from base stations to the target source can be measured as $\theta_{i}\left(0 \leq \theta_{i} \leq \frac{\pi}{2}\right)$. On this basis, the distance $d_{\alpha i}$ between projections of base stations and the target source on the $\alpha$ plane can be obtained as $d_{\alpha i}=d_{i} \times \sin \theta_{i}$. Thus, the equation system is as follows:

$$
\left\{\begin{array}{c}
\left(x_{\alpha 1}-x_{\alpha T}\right)^{2}+\left(y_{\alpha 1}-y_{\alpha T}\right)^{2}=d_{\alpha 1}{ }^{2} \sin ^{2} \theta_{1} \\
\left(x_{\alpha 2}-x_{\alpha}\right)^{2}+\left(y_{\alpha 2}-y_{\alpha}\right)^{2}=d_{\alpha 2}{ }^{2} \sin ^{2} \theta_{2} \\
\vdots \\
\left(x_{\alpha i}-x_{\alpha}\right)^{2}+\left(y_{\alpha i}-y_{\alpha}\right)^{2}=d_{\alpha i}{ }^{2} \sin ^{2} \theta_{i} \\
\vdots \\
\left(x_{\alpha n}-x_{\alpha}\right)^{2}+\left(y_{\alpha n}-y_{\alpha}\right)^{2}=d_{\alpha n}{ }^{2} \sin ^{2} \theta_{n}
\end{array}\right.
$$

The projection coordinates of the target source on $\mathrm{XY}$ plane $\left(x_{\alpha T}, y_{\alpha T}\right)$ are calculated by the maximum likelihood method [25]. The specific process is as follows:

In this equation system, the terms from 1 to $(n-1)$ are subtracted by term $n$; hence: 
$\left\{\begin{array}{c}x_{\alpha 1}{ }^{2}-x_{\alpha n}{ }^{2}-2\left(x_{\alpha 1}-x_{\alpha n}\right) x_{\alpha T}+y_{\alpha 1}{ }^{2}-y_{\alpha n}{ }^{2}-2\left(y_{\alpha 1}-y_{\alpha n}\right) y_{\alpha T}=d_{\alpha 1}{ }^{2} \sin ^{2} \theta_{1}-d_{\alpha n}{ }^{2} \sin ^{2} \theta_{n} \\ x_{\alpha 2}{ }^{2}-x_{\alpha n}{ }^{2}-2\left(x_{\alpha 2}-x_{\alpha n}\right) x_{\alpha T}+y_{\alpha 2}{ }^{2}-y_{\alpha n}{ }^{2}-2\left(y_{\alpha 2}-y_{\alpha n}\right) y_{\alpha T}=d_{\alpha 2}{ }^{2} \sin ^{2} \theta_{2}-d_{\alpha n}{ }^{2} \sin ^{2} \theta_{n} \\ \vdots \\ x_{\alpha i}{ }^{2}-x_{\alpha n}{ }^{2}-2\left(x_{\alpha i}-x_{\alpha n}\right) x_{\alpha T}+y_{\alpha i}{ }^{2}-y_{\alpha n}{ }^{2}-2\left(y_{\alpha i}-y_{\alpha n}\right) y_{\alpha T}=d_{\alpha i}{ }^{2} \sin ^{2} \theta_{i}-d_{\alpha n}{ }^{2} \sin ^{2} \theta_{n} \\ \vdots \\ x_{\alpha(n-1)}{ }^{2}-x_{\alpha n}{ }^{2}-2\left(x_{\alpha(n-1)}-x_{\alpha n}\right) x_{\alpha T}+y_{\alpha(n-1)}{ }^{2}-y_{\alpha n}{ }^{2}-2\left(y_{\alpha(n-1)}-y_{\alpha n}\right) y_{\alpha T}=d_{\alpha(n-1)}{ }^{2} \sin ^{2} \theta_{(n-1)}-d_{\alpha n}{ }^{2} \sin ^{2} \theta_{n}\end{array}\right.$

Let $X=\left(x_{\alpha T}, y_{\alpha T}\right)^{T}$. Therefore, the above equation system can be transformed into a linear expression of $A x=b$, where:

$$
A=\left[\begin{array}{cc}
2\left(x_{\alpha 1}-x_{\alpha n}\right) & 2\left(y_{\alpha 1}-y_{\alpha n}\right) \\
2\left(x_{\alpha 2}-x_{\alpha n}\right) & 2\left(y_{\alpha 2}-y_{\alpha n}\right) \\
\vdots & \vdots \\
2\left(x_{\alpha(n-1)}-x_{\alpha n}\right) & 2\left(y_{\alpha(n-1)}-y_{\alpha n}\right)
\end{array}\right]
$$

$$
b=\left[\begin{array}{c}
x_{\alpha 1}{ }^{2}-x_{\alpha n}{ }^{2}+y_{\alpha 1}{ }^{2}-y_{\alpha n}{ }^{2}+d_{\alpha n}{ }^{2} \sin ^{2} \theta_{n}-d_{\alpha 1}{ }^{2} \sin ^{2} \theta_{1} \\
x_{\alpha 2}{ }^{2}-x_{\alpha n}{ }^{2}+y_{\alpha 2}{ }^{2}-y_{\alpha n}{ }^{2}+d_{\alpha n}{ }^{2} \sin ^{2} \theta_{n}-d_{\alpha 2}{ }^{2} \sin ^{2} \theta_{2} \\
\vdots \\
x_{\alpha i}{ }^{2}-x_{\alpha n}{ }^{2}+y_{\alpha i}{ }^{2}-y_{\alpha n}{ }^{2}+d_{\alpha n}{ }^{2} \sin ^{2} \theta_{n}-d_{\alpha i}{ }^{2} \sin ^{2} \theta_{i} \\
\vdots \\
x_{\alpha(n-1)}{ }^{2}-x_{\alpha n}{ }^{2}+y_{\alpha(n-1)}{ }^{2}-y_{\alpha n}{ }^{2}+d_{\alpha n}{ }^{2} \sin ^{2} \theta_{n}-d_{\alpha(n-1)}{ }^{2} \sin ^{2} \theta_{(n-1)}
\end{array}\right]
$$

angle of pitch and horizontal angle is used as the final

Based on the least squares, $\hat{X}=\left(A^{\mathrm{T}} A\right)^{-1} A^{\mathrm{T}} b$.

The projection coordinates of the target source in the XY plane can be obtained as $\left(x_{\alpha T}, y_{\alpha T}\right)$.

The horizontal angle $\sigma_{i}\left(0 \leq \sigma_{i} \leq \frac{\pi}{2}\right)$ of signals from base stations to the target source can be also measured. The projection coordinates of the target source on the $\mathrm{XY}$ coordinate plane are calculated based on the distance $d_{i}$ between the base stations and target source. If the $\mathrm{XZ}$ coordinate plane is the $\beta$ plane, then the projection distance between the base stations and target source in the $\beta$ plane is $d_{\beta i}=d_{i} \times \sin \sigma_{i}$. The projection coordinates of the target source in this plane are $\left(x_{\beta T}, z_{\beta T}\right)$.

In the above calculation method of coordinates of the target source, the distance between the target source and base stations and the angle of pitch and horizontal angle for the target source receiving signals from base stations must be calculated. Measuring error is inevitable due to environmental interferences. A large number of interference factors indicate large measuring error and low positioning accuracy. Therefore, the two groups of x-coordinates cannot be calculated by simply using the averaging method. Accurate data will be obtained by calculating according to their weights.

\subsection{D plane coordinates of weighting fusion}

The measurement distance between the target source and base stations must be similar when calculating the two groups of $\mathrm{x}$-coordinates. Measurement accuracy is determined by the accuracy of angle of pitch and horizontal angle. Large angle causes large measuring error. Angle is proportional to its sine and inversely proportional to accuracy. Therefore, the reciprocal sum of the sine values of weight in this study.

The weight of $x_{\alpha T}$ in $\alpha$ plane is as follows:

$$
Q_{\alpha}=\frac{\sum_{i=1}^{n} \frac{1}{\sin \theta_{i}}}{\sum_{i=1}^{n}\left(\frac{1}{\sin \theta_{i}}+\frac{1}{\sin \sigma_{i}}\right)}
$$

The weight of $x_{\beta T}$ in $\beta$ plane is as follows:

$$
Q_{\beta}=\frac{\sum_{i=1}^{n} \frac{1}{\sin \sigma_{i}}}{\sum_{i=1}^{n}\left(\frac{1}{\sin \theta_{i}}+\frac{1}{\sin \sigma_{i}}\right)}
$$

Finally, the measurement coordinates of the target source can be obtained as:

$$
\begin{aligned}
& x_{T}=Q_{\alpha} x_{\alpha T} \\
& =\frac{\sum_{i=1}^{n} \frac{1}{\sin \theta_{i}}}{\sum_{i=1}^{n}\left(\frac{1}{\sin \theta_{i}}+\frac{1}{\sin \sigma_{i}}\right)} x_{\alpha T}+\frac{\sum_{i=1}^{n} \frac{1}{\sin \sigma_{i}}}{\sum_{i=1}^{n}\left(\frac{1}{\sin \theta_{i}}+\frac{1}{\sin \sigma_{i}}\right)} x_{\beta T}
\end{aligned}
$$

If the included angle between the signal emission direction and $\mathrm{YZ}$ plane (named as $\gamma$ plane) is $\varphi\left(0 \leq \sigma_{i} \leq \frac{\pi}{2}\right)$, then three groups of $2 \mathrm{D}$ position coordinates can be acquired as $\left(x_{\alpha T}, y_{\alpha T}\right),\left(x_{\beta T}, z_{\beta T}\right)$, and $\left(y_{\gamma T}, z_{\gamma T}\right)$. 
Then, the weights of $y_{T}$ in $\alpha$ plane and $\gamma$ plane are as follows:

$$
\left\{\begin{array}{c}
Q_{\alpha}=\frac{\sum_{i=1}^{n} \frac{1}{\sin \theta_{i}}}{\sum_{i=1}^{n}\left(\frac{1}{\sin \theta_{i}}+\frac{1}{\sin \left(\frac{\pi}{2}-\varphi_{i}\right)}\right)} \\
Q_{\gamma}=\frac{\sum_{i=1}^{n} \frac{1}{\sin \left(\frac{\pi}{2}-\varphi_{i}\right)}}{\sum_{i=1}^{n}\left[\frac{1}{\sin \theta_{i}}+\frac{1}{\sin \left(\frac{\pi}{2}-\varphi_{i}\right)}\right]}
\end{array}\right.
$$

The weights of $z_{T}$ in $\beta$ plane and $\gamma$ plane are as follows:

$$
\left\{\begin{array}{c}
Q_{\beta}=\frac{\sum_{i=1}^{n} \frac{1}{\sin \sigma_{i}}}{\sum_{i=1}^{n}\left(\frac{1}{\sin \sigma_{i}}+\frac{1}{\sin \left(\frac{\pi}{2}-\varphi_{i}\right)}\right)} \\
Q_{\gamma}=\frac{\sum_{i=1}^{n} \frac{1}{\sin \left(\frac{\pi}{2}-\varphi_{i}\right)}}{\sum_{i=1}^{n}\left[\frac{1}{\sin \sigma_{i}}+\frac{1}{\sin \left(\frac{\pi}{2}-\varphi_{i}\right)}\right]}
\end{array}\right.
$$

The 3D position coordinates of the target source under this circumstance can be expressed as:

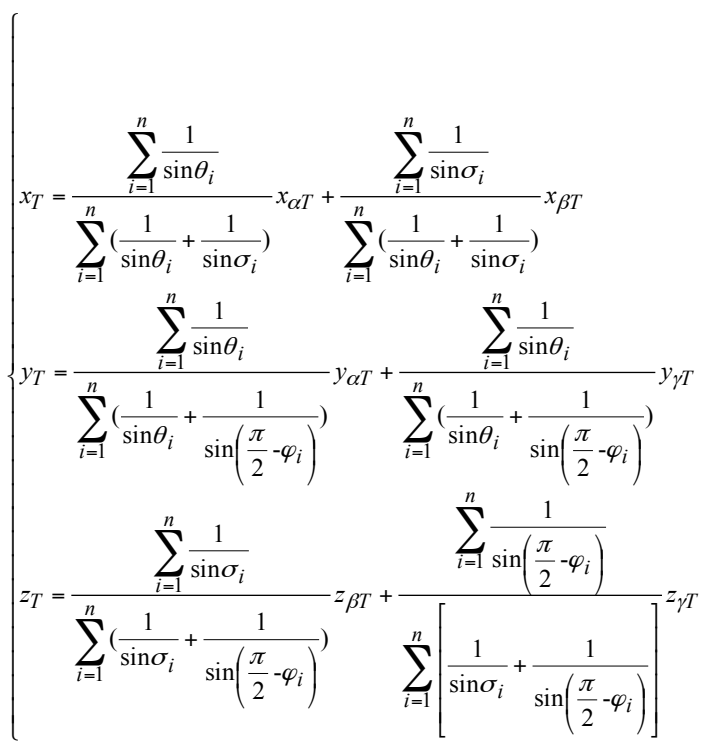

\subsection{Algorithm flow}

First, the proposed algorithm acquires the position coordinates of multiple groups of target sources in the $2 \mathrm{D}$ plane by using maximum likelihood estimation with TDOA and AOA of the signals from the base stations to the target source. Second, weighting fusion is conducted to obtain the $3 \mathrm{D}$ position coordinates according to the relative angle between the signals emitted from the base stations and target source.

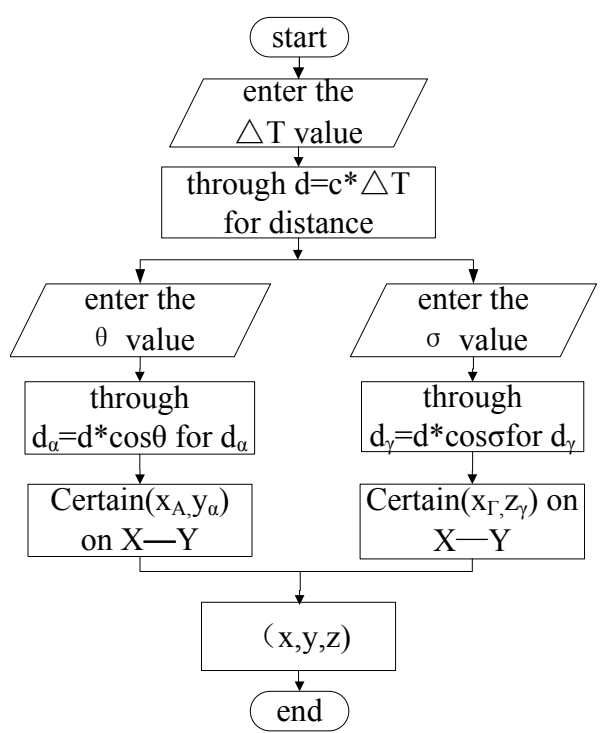

Fig. 2. Algorithm flowchart

\section{Results and Discussion}

\subsection{Experimental analysis of feasibility}

One $500 \mathrm{~m} \times 500 \mathrm{~m} \times 100 \mathrm{~m}$ spatial coordinate system was established. In this space, 10 targets were distributed randomly, and three base stations were set (Fig.3). One of base station was determined as the origin of coordinate system. The coordinates of the three base stations were $(0,0,0),(250,300,0)$ and $(150,100,50)$, respectively. The signal propagation velocity was $c=3 \times 10^{8} \mathrm{~m} / \mathrm{s}$.

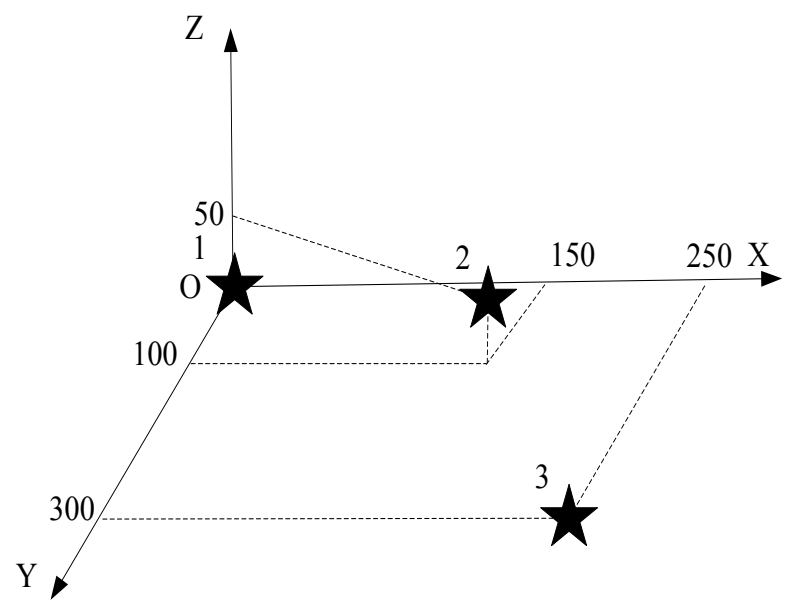

Fig. 3. Layout of base stations

The coordinate's errors of the above algorithm are shown in Table 1.

Table 1 shows that the mean position error of the proposed algorithm could be calculated $0.243 \mathrm{~m}$ based on the actual and measured coordinates of the 10 testing points, the actual and measured distances between the testing points to base stations, and the actual and measured AOA values. Matlab 3D simulation of the above data was conducted. Distributions of actual and measured coordinates of the 10 
testing points in the space are shown in Fig.4. The proposed algorithm had small error in the $500 \mathrm{~m} \times 500 \mathrm{~m} \times 100 \mathrm{~m}$

coordinate space.

Table 1. Comparison between the actual and measurement data in the simulation experiment and measurement errors

\begin{tabular}{|c|c|c|c|c|c|c|c|c|c|}
\hline \multirow[t]{3}{*}{ No. } & \multicolumn{2}{|c|}{$\begin{array}{c}\text { Coordinates of the target } \\
\text { source }\end{array}$} & \multicolumn{2}{|c|}{$\begin{array}{c}\text { Distance between the } \\
\text { target source and base } \\
\text { stations }\end{array}$} & \multicolumn{4}{|c|}{ Angle of the target source of base stations } & \multirow[t]{3}{*}{$\begin{array}{l}\text { Measuremen } \\
t \text { error of the } \\
\text { target source }\end{array}$} \\
\hline & \multirow[t]{2}{*}{ Actual } & \multirow{2}{*}{ Measurement } & \multirow[t]{2}{*}{ Actual } & \multirow{2}{*}{ Measurement } & \multicolumn{2}{|r|}{$\theta$} & \multicolumn{2}{|r|}{$\sigma$} & \\
\hline & & & & & Actual & Measurement & Actual & Measurement & \\
\hline \multirow[t]{3}{*}{1} & 212 & 211.95 & 395.10 & 395.08 & 82.14 & 82.14 & 57.55 & 57.56 & \multirow[t]{3}{*}{0.065} \\
\hline & 329 & 329.01 & 72.12 & 72.18 & 41.52 & 41.52 & 58.20 & 58.16 & \\
\hline & 54 & 54.04 & 237.28 & 237.28 & 89.03 & 89.03 & 74.85 & 74.87 & \\
\hline \multirow[t]{3}{*}{2} & 417 & 417.02 & 429.87 & 429.82 & 85.20 & 85.19 & 14.06 & 14.02 & \multirow[t]{3}{*}{0.045} \\
\hline & 98 & 98.00 & 264.55 & 264.88 & 82.18 & 82.18 & 50.86 & 50.91 & \\
\hline & 36 & 36.04 & 267.37 & 266.67 & 87.00 & 87.00 & 3.03 & 4.00 & \\
\hline \multirow[t]{3}{*}{3} & 136 & 136.00 & 284.68 & 284.69 & 88.59 & 88.59 & 61.46 & 61.46 & \multirow[t]{3}{*}{0.014} \\
\hline & 250 & 250.01 & 124.68 & 124.68 & 86.78 & 86.78 & 23.89 & 23.89 & \\
\hline & 7 & 7.01 & 156.67 & 156.68 & 74.07 & 74.07 & 84.87 & 84.87 & \\
\hline \multirow[t]{3}{*}{4} & 89 & 88.70 & 237.70 & 237.59 & 72.87 & 72.86 & 68.01 & 68.08 & \multirow[t]{3}{*}{0.300} \\
\hline & 209 & 209.00 & 197.74 & 197.99 & 69.27 & 69.30 & 35.49 & 35.34 & \\
\hline & 70 & 70.01 & 126.50 & 126.65 & 80.90 & 80.91 & 61.17 & 61.05 & \\
\hline \multirow[t]{3}{*}{5} & 78 & 78.02 & 106.46 & 106.49 & 52.37 & 52.40 & 42.89 & 42.89 & \multirow[t]{3}{*}{0.135} \\
\hline & 32 & 32.13 & 325.01 & 325.71 & 78.46 & 78.49 & 58.05 & 58.13 & \\
\hline & 65 & 64.97 & 100.16 & 100.06 & 81.39 & 81.40 & 44.04 & 44.00 & \\
\hline \multirow[t]{3}{*}{6} & 431 & 431.16 & 571.66 & 571.79 & 86.59 & 86.60 & 41.07 & 41.06 & \multirow[t]{3}{*}{0.196} \\
\hline & 374 & 374.03 & 198.48 & 198.62 & 80.14 & 80.18 & 24.22 & 24.20 & \\
\hline & 34 & 33.89 & 392.80 & 392.94 & 87.67 & 87.65 & 44.33 & 44.31 & \\
\hline \multirow[t]{3}{*}{7} & 263 & 263.01 & 299.60 & 299.63 & 87.70 & 87.70 & 28.62 & 28.62 & \multirow[t]{3}{*}{0.032} \\
\hline & 143 & 143.03 & 157.99 & 157.96 & 85.64 & 85.64 & 85.28 & 85.28 & \\
\hline & 12 & 12.00 & 126.74 & 126.76 & 72.55 & 72.56 & 26.92 & 26.93 & \\
\hline \multirow[t]{3}{*}{8} & 357 & 355.80 & 357.94 & 356.74 & 88.08 & 88.07 & 4.16 & 4.16 & \multirow[t]{3}{*}{1.200} \\
\hline & 23 & 23.00 & 297.19 & 296.79 & 87.69 & 87.68 & 68.90 & 69.12 & \\
\hline & 12 & 12.00 & 224.10 & 222.99 & 80.24 & 80.19 & 22.53 & 22.64 & \\
\hline 9 & 125 & 124.96 & 266.29 & 266.17 & 85.05 & 85.04 & 62.00 & 62.00 & 0.128 \\
\hline & 234 & 233.88 & 143.21 & 143.31 & 80.76 & 80.76 & 29.21 & 29.25 & \\
\hline & 23 & 23.02 & 138.96 & 138.85 & 78.80 & 78.80 & 79.64 & 79.61 & \\
\hline 10 & 156 & 156.03 & 383.70 & 383.99 & 78.42 & 78.43 & 66.01 & 66.02 & 0.311 \\
\hline & 342 & 342.31 & 128.57 & 128.64 & 53.21 & 53.23 & 43.02 & 43.03 & \\
\hline & 77 & 77.00 & 243.58 & 243.88 & 83.64 & 83.64 & 88.59 & 88.58 & \\
\hline & & & & Mean valu & & & & & 0.243 \\
\hline
\end{tabular}

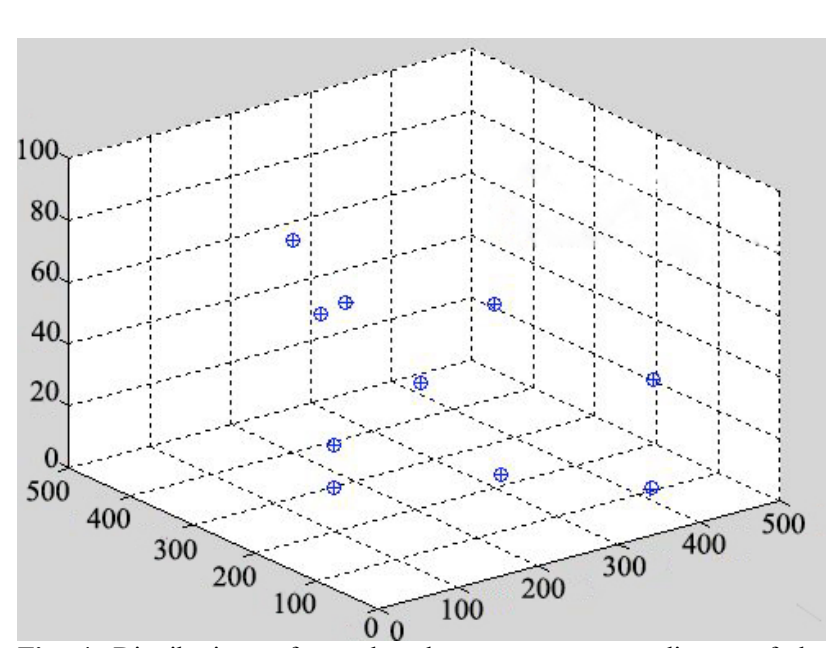

Fig. 4. Distributions of actual and measurement coordinates of the testing points in space

Note: -+- represents Actual coordinate; $-\mathrm{o}-$ represents Measurement coordinate.

\subsection{Contrast experiment}

Wireless localization generally uses TDOA, AOA, or TDOA-AOA combined algorithms. The proposed algorithm was compared with the above two algorithms. The TDOA localization algorithm set up the following equation system by measuring the time difference when the target source receives the signals from different base stations to calculate the position coordinates of the target source.

$$
\left\{\begin{array}{l}
\sqrt{\left(x_{1}-x\right)^{2}+\left(y_{1}-y\right)^{2}+\left(z_{1}-z\right)^{2}}-\sqrt{\left(x_{0}-x\right)^{2}+\left(y_{0}-y\right)^{2}+\left(z_{0}-z\right)^{2}} \\
=c \times \Delta t_{1} \\
\sqrt{\left(x_{2}-x\right)^{2}+\left(y_{2}-y\right)^{2}+\left(z_{2}-z\right)^{2}-\sqrt{\left(x_{0}-x\right)^{2}+\left(y_{0}-y\right)^{2}+\left(z_{0}-z\right)^{2}}} \\
=c \times \Delta t_{2} \\
\sqrt{\left(x_{3}-x\right)^{2}+\left(y_{3}-y\right)^{2}+\left(z_{3}-z\right)^{2}}-\sqrt{\left(x_{0}-x\right)^{2}+\left(y_{0}-y\right)^{2}+\left(z_{0}-z\right)^{2}} \\
=c \times \Delta t_{3}
\end{array}\right.
$$

Where $\left(x_{0}, y_{0}, z_{0}\right)$ is the position coordinates of the reference base station, $\left(x_{i}, y_{i}, z_{i}\right)$ is the position coordinates of measuring base stations, $c$ is signal propagation velocity, and $\Delta t_{i}$ is the time differences of arrival to the target between the reference base station and base station $i$.

According to the measurement values, the TDOA-AOA combined algorithm set up the following equation system:

$$
\left\{\begin{array}{l}
\sqrt{\left(x_{1}-x\right)^{2}+\left(y_{1}-y\right)^{2}}=c \times T_{1} \times \sin \theta_{1} \\
\sqrt{\left(x_{2}-x\right)^{2}+\left(y_{2}-y\right)^{2}}=c \times T_{2} \times \sin \theta_{2} \\
\sqrt{\left(x_{3}-x\right)^{2}+\left(y_{3}-y\right)^{2}}=c \times T_{2} \times \sin \theta_{2}
\end{array}\right.
$$


Where $T_{i}$ is TOA of signal from base station $i$ to the target source and $\theta_{i}$ is the angle of pitch of the signal from base station $i$ to the target source. Thus, the coordinates of the target source $(x, y)$ could be calculated from Equation (13). The mean of the target source in the $z$ coordinate system could be calculated by the following equation:

$\left|z_{i}-z\right|=c \times T_{i} \cos \alpha$

These two localization algorithms were compared with the proposed algorithm under same experimental conditions. Positioning accuracies of three algorithms are shown in Table 2.

The corresponding position coordinates are listed in Table 2.

Tab. 2. Coordinates and error of testing points by different localization algorithms

\begin{tabular}{|c|c|c|}
\hline No. & TDOA & TDOA+AOA \\
\hline \multirow[t]{3}{*}{1} & 212.71 & 212.47 \\
\hline & 330.14 & 329.83 \\
\hline & 52.68 & 53.97 \\
\hline \multirow[t]{3}{*}{2} & 415.23 & 417.58 \\
\hline & 100.16 & 97.63 \\
\hline & 37.22 & 35.47 \\
\hline \multirow[t]{3}{*}{3} & 134.67 & 136.81 \\
\hline & 250.91 & 250.27 \\
\hline & 7.03 & 7.01 \\
\hline \multirow[t]{3}{*}{4} & 88.56 & 89.43 \\
\hline & 209.71 & 210.37 \\
\hline & 69.23 & 69.76 \\
\hline \multirow[t]{3}{*}{5} & 77.41 & 78.21 \\
\hline & 32.07 & 32.11 \\
\hline & 64.50 & 65.07 \\
\hline \multirow[t]{3}{*}{6} & 433.15 & 430.72 \\
\hline & 373.24 & 374.58 \\
\hline & 34.61 & 33.83 \\
\hline \multirow[t]{3}{*}{7} & 264.47 & 263.84 \\
\hline & 141.75 & 144.03 \\
\hline & 12.03 & 12.01 \\
\hline \multirow[t]{3}{*}{8} & 357.92 & 356.41 \\
\hline & 23.03 & 23.04 \\
\hline & 12.02 & 12.02 \\
\hline \multirow[t]{3}{*}{9} & 124.38 & 125.64 \\
\hline & 235.10 & 234.43 \\
\hline & 22.31 & 23.04 \\
\hline \multirow[t]{3}{*}{10} & 154.37 & 156.32 \\
\hline & 340.22 & 342.16 \\
\hline & 77.08 & 76.98 \\
\hline
\end{tabular}

Positioning errors of three localization algorithms are shown in Fig.5.

The coordinate errors of TDOA localization algorithm and TDOA-AOA combined localization algorithm were compared. Table 2 shows that the TDOA-AOA combined localization algorithm could effectively increase the localization accuracy. Comparison of data in Table 1 and Table 2 showed that the $x$ value calculated from weighting was significantly more accurate than the value calculated by averaging method. The proposed algorithm increased the positioning accuracy of target source by $48.27 \%$ and $68.33 \%$ of those from TDOA-AOA combined algorithm and TDOA algorithm, respectively.

\section{Conclusions}

This study analyzed the effects of the number of base stations and computation complexity on positioning accuracy and discussed the weights of measurement results of different base stations to increase wireless positioning accuracy and reduce the complexity of localization algorithm under multiple base stations $(>4)$. The following conclusions could be drawn:

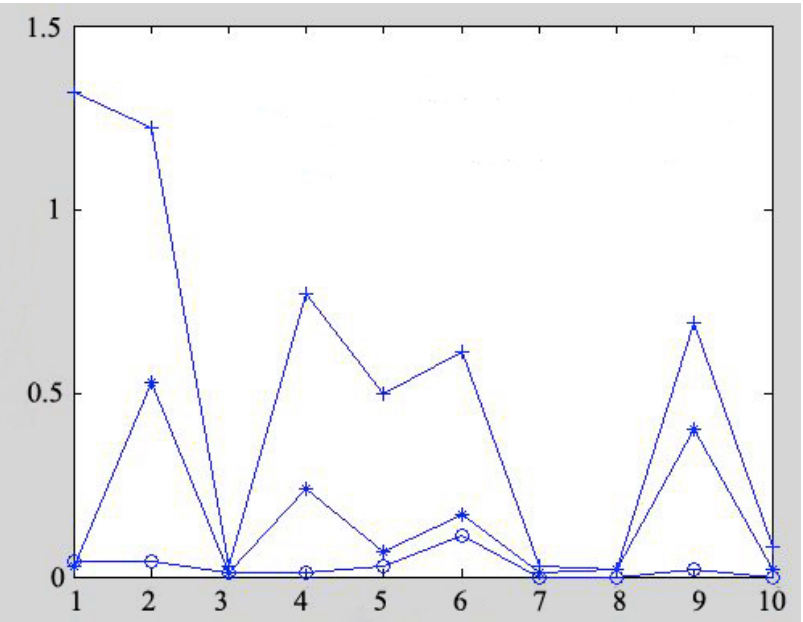

Fig. 5. Comparison of three localization algorithms in coordinate errors Note: -+- represents TDOA measurement error; - *- represents TDOA+AOA measurement error; - $\mathrm{O}-$ represents algorithm based on weight fusion of target position coordinates measurement error.

(1) During localization, increasing the number of base stations can significantly increase the positioning accuracy of the target source and prevent accidental damages. The number of base station is proportional to positioning accuracy. At least four base stations can be set if allowed by the conditions and costs.

(2) Weight of base station is determined by the measurement error of the base station. In the proposed algorithm, the relative angle between the base stations and target source determines the weight of measurement data. According to analysis, the sine of angle is inversely proportional to positioning accuracy.

Therefore, the proposed algorithm can effectively reduce the complexity caused by increasing the number of base stations, improve the localization efficiency in engineering practices, integrate the $2 \mathrm{D}$ coordinates into $3 \mathrm{D}$ coordinates according to different weights, and significantly increase the positioning accuracy as compared with averaging method. Moreover, this method can be used in situations with a relatively large number of base stations to enhance the damage resistance of the positioning system. However, solving the target projection coordinates in the $\mathrm{YZ}$ plane is difficult because base stations can only measure the angle of pitch and horizontal angle of transmitted signals during angle measurement. Thus, the data of weighting fusion are limited, and the positioning accuracy is reduced. In future studies, the angle measurement functions of base stations must be increased to increase available data and thereby improve the target positioning accuracy.

This is an Open Access article distributed under the terms of the Creative Commons Attribution Licence

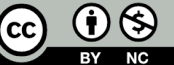




\section{References}

1. Guan, W. G., Gao, Y., Yao, Q. Z., “TDOA location algorithm based on best linear unbiased estimation". Application Research of Computers, 32(8), 2015, pp. 2472-2474.

2. Ibrahim, N., Safitri, I., Hamid, E. Y., "3D localization technique for broad band impulsive noise source". In: International Conference on Computer, Sydney, Australia: IEEE, 2014, pp. 99-104.

3. Miyazaki, H., "An Improvement Measure for 3D TDOA Localization by Using Height Information". Review of Scientific Instruments, 79(10), 2015, pp. 10E533-10E533-3.

4. Bensky, A., "Wireless positioning technologies and applications". Global Positioning System, 9(6), 2008, pp. 218-228.

5. Ssu, K. F., Ou, C. H., Jiau, H C., "Localization with mobile anchor points in wireless sensor networks". IEEE Transactions on Vehicular Technology, 54 (3), 2005, pp. 1187-1197.

6. Bishop, A., Anderson, B., Pathirana, P., "Exploiting geometry for improved hybrid AOA/TDOA-based localization". Signal Processing, 88(7), 2008, pp. 1775-1791.

7. Ferreol, A., Bosse, J., Larzabal, P., "A low-cost AOA-TDOA approach for blind geolocation in multi-paths context". In: European Signal Processing Conference, Aalborg, Denmark: IEEE, 2010, pp. 686-690.

8. Liu, C., Yang, J., Wang, F., "Joint TDOA and AOA location algorithm". Journal of Systems Engineering \& Electronics, 24(2) 2013, pp. 183-188.

9. Zhang, X., Sihong, X. T., Hong, T. Y., “An integrated closed-form fusion algorithm for TDOA/AOA". In: International Conference on Information Fusion, Heidelberg, Germany: IEEE, 88(7), 2016, pp. 5-8.

10. Giacometti, R., Baussard, A., Jahan, D., "Localization of radar emitters from a single sensor using multipath and TDOA-AOA measurements in a naval context". In: Signal Processing Conference, Hilton Budapest, Hungary: IEEE, 2016, pp. 692-696.

11. Shikur, B. Y., Weber, T., "TDOA/AOD/AOA localization in NLOS environments". In: International Conference on Acoustics, Speech and Signal Processing, Florence, Italy: IEEE, 2014, pp. 6518-6522.

12. Shikur, B. Y., Weber, T., "Posterior CRLB for tracking a mobile station in NLOS multipath environments". In: International Conference on Acoustics, Speech and Signal Processing, Vancouver, Canada: IEEE, 2013, pp. 5175-5179.

13. Giacometti, R., Baussard, A., Cornu, C., "Accuracy studies for TDOA-AOA localization of emitters with a single sensor". In: Radar Conference, Philadelphia, USA: IEEE, 2016, pp. 1-4.
14. Lim, D. W., Kang, J. M., Heo, M. B., "Hybrid TDOA/AOA Localization Algorithm for GPS Jammers". Journal of Institute of Control, 20(1), 2014, pp. 101-105.

15. Lim, D. W., Kang, H. W., Sang, J. L., \& Hwang, D. H., "Position dop analysis for sensor placement in the tdoa-based localization system". Journal of Electrical Engineering \& Technology, 7(6), 2012, pp. 1009-1013.,

16. Lei, W. Y., Chen, B. X., Yang, M. L., \& Zhu, W., "Passive 3d target location method based on TOA and TDOA". Systems Engineering and Electronics, 36(5), 2014, pp. 816-823.

17. Kim, D. K., Ha, J. H., Kim, P. J., "TDOA/AOA localization in RFID system using dual indirect Kalman filter". IEEE/SICE International Symposium on System Integration, 2011, pp. 440-445.

18. Choi, H. H., Jin, M. H., Lim, D. W., "A Study of DOA estimation based on TDOA/AOA for jammer localization". Journal of Korea Navigation Institute, 15(6), 2011, pp. 962-969.

19. Alarifi, A., Al-Salman, A., Alsaleh, M., "Ultra Wideband Indoor Positioning Technologies: Analysis and Recent Advances". Sensors, 16(5), 2016, pp. 1-36.

20. Anumba, C. J., Wang, X., "Ubiquitous User Localization for Pervasive Context-Aware Construction Applications". Wiley Blackwell, 31(4), 2012, pp. 391-402.

21. Tang, H., Park, Y., Qiu, T., "A TOA-AOA-Based NLOS Error Mitigation Method for Location Estimation". Eurasip Journal on Advances in Signal Processing, 2008(1), pp. 1-14.

22. Tang, H., Park, Y., Qiu, T., "NLOS mitigation for TOA location based on a modified deterministic model". Journal of Electrical \& Computer Engineering, 2008(1), pp. 2-13.

23. Shin, D. H., Sung, T. K., "Comparisons of error characteristics between TOA and TDOA positioning". Aerospace \& Electronic Systems, 38(1), 2009, pp. 307-311.

24. Cong, L., Zhuang, W., "Non-line-of-sight error mitigation in TDOA mobile location". IEEE Transactions on Wireless Communications, 4(2), 2001, pp. 560-573.

25. Prorok, A., Gonon, L., Martinoli, A., "Online model estimation of ultra-wideband TDOA measurements for mobile robot localization". In: International Conference on Robotics and Automation, St. Paul, USA: IEEE, 2012, pp. 807-814 